\title{
Cytotoxic effects and apoptotic signalling mechanisms of the sesquiterpenoid euplotin $C$, a secondary metabolite of the marine ciliate Euplotes crassus, in tumour cells
}

\author{
D. Cervia, D. Martini, M. Garcia-Gil, G. Di Giuseppe, G. Guella, F. Dini and P. Bagnoli \\ Dipartimento di Fisiologia e Biochimica "G. Moruzzi”, via S. Zeno, 56127 Pisa, Italy (D. Cervia, D. Martini, M. Garcia-Gil, P. \\ Bagnoli); Dipartimento di Etologia, Ecologia ed Evoluzione, Università di Pisa, 56127, Pisa, Italy (G. Di Giuseppe, F. Dini); \\ Dipartimento di Fisica, Laboratorio di Chimica bioorganica, Università di Trento, 38050 Trento, Italy (G. Guella)
}

Published online: 9 March 2006

Most antitumour agents with cytotoxic properties induce apoptosis. The lipophilic compound euplotin $\mathbf{C}$, isolated from the ciliate Euplotes crassus, is toxic to a number of different opportunistic or pathogenic microorganisms, although its mechanism of action is currently unknown. We report here that euplotin $C$ is a powerful cytotoxic and pro-apoptotic agent in mouse AtT-20 and rat PC12 tumour-derived cell lines. In addition, we provide evidence that euplotin $\mathrm{C}$ treatment results in rapid activation of ryanodine receptors, depletion of $\mathrm{Ca}^{2+}$ stores in the endoplasmic reticulum (ER), the release of cytochrome $c$ from the mitochondria, activation of caspase-12, and activation of caspase-3, leading to apoptosis. Intracellular $\mathrm{Ca}^{2+}$ overload is an early event which induces apoptosis and is parallelled by ER stress and the release of cytochrome $c$, whereas caspase-12 may be activated by euplotin $C$ at a later stage in the apoptosis pathway. These events, either independently or concomitantly, lead to the activation of the caspase-3 and its downstream effectors, triggering the cell to undergo apoptosis. These results demonstrate that euplotin $\mathbf{C}$ may be considered for the design of cytotoxic and pro-apoptotic new drugs.

Keywords: $\mathrm{Ca}^{2+}$ stores; caspases; cell death; cytochrome c; endoplasmic reticulum.

\section{Introduction}

Finely tuned regulation of intracellular signalling is fundamental for cell survival and death. In particular, cell demise does not always result from a collapse of homeostatic control systems but is often an active and highly regulated process. ${ }^{1-5}$ Cell death can be induced by two mechanisms: necrosis and apoptosis. Necrosis has traditionally been thought of as simply a passive, unorganized form of cell death whereas apoptosis is a more programmed process and is the principal mechanism by which cells are physiologically eliminated. Alternatively, non-apoptotic forms of

Correspondence to: D. Cervia, Dipartimento di Fisiologia e Biochimica "G. Moruzzi", via S. Zeno, 56127 Pisa, Italy. Tel.: +39-0502213459; Fax: +39-050-2213527; e-mail: d.cervia@dfb.unipi.it programmed cell death have been recently described and classified as programmed necrosis or autophagic cell death. ${ }^{5}$ Apoptosis is an intrinsic cellular suicide mechanism and is a relatively linear process. It is triggered by an initiation phase that is highly varied depending on cell type and the underlying stimulus. This is followed by an effector phase where the cell undergoes distinct biochemical changes that result in the systematic activation of catabolic enzymes (proteases and/or nucleases) that ultimately cause the cleavage of proteins and DNA. ${ }^{3,4}$ Induction of apoptosis is arguably the body's most potent defence against cancer. Indeed, immune system cells destroy cancerous cells, and most chemotherapeutic and chemopreventive agents inhibit tumour cell proliferation, by inducing apoptosis. ${ }^{6-9}$ Unfortunately, these agents are of limited use for clinical applications owing to their inherent lack of stability, specificity, limited solubility, and poor cell permeability. ${ }^{9}$ Currently, a great deal of effort is being aimed at replacing the existing drugs by novel compounds targeting specific apoptosis regulators, which could set the stage for future therapeutics.

Cellular apoptosis is ultimately caused by the activation of caspases (cysteine proteases). Active initiator caspases can proteolyze additional executioner caspases, for instance caspase- 3 , generating a caspase cascade that cleaves proteins critical for cell survival. Currently a number of different mechanisms of apoptosis have been characterised which may overlap due to their cross-talk and which may or may not be associated with caspase activation. ${ }^{1,2,6,7}$ Recent developments have emphasized the central role of $\mathrm{Ca}^{2+}$ in cell death pathways. Indeed, $\mathrm{Ca}^{2+}$ depletion from intracellular stores can activate distinct stages of the cell death programme, which can then function alone or in conjunction with other sub-programmes to kill the cell. ${ }^{2}$ Growing evidence suggests that, in addition to the injured mitochondria which release cytochrome $c$ and other apoptogenic factors, other organelles are also major points of integration for proapoptotic signalling and caspase activation. ${ }^{1}$ For instance, the endoplasmic reticulum (ER) participates in the initiation of apoptosis through at least two different mechanisms, namely the unfolded protein response and $\mathrm{Ca}^{2+}$ signalling 